\author{
Suzanna Lundblad ${ }^{1,2}$, Danilo Garcia ${ }^{3,4}$, \\ Berit Hansson ${ }^{1}$ and Trevor Archer ${ }^{2,4 *}$ \\ ${ }^{1}$ Sahlgrenska University Hospital, Affektiva II \\ Anorexia \& Bulimia Clinic for Adults, S 41666 \\ Gothenburg, Sweden \\ ${ }^{2}$ University of Gothenburg, Department of \\ Psychology, Gothenburg, Sweden \\ ${ }^{3}$ Instutute of Neuroscience and Physiology, Centre \\ for Ethics, Law and Mental Health (CELAM), \\ University of Gothenburg, Gothenburg, Sweden \\ ${ }^{4}$ Network for Empowerment and Well-being \\ Dates: Received: 25 May, 2015; Accepted: 10 \\ June, 2015; Published: 12 June, 2015 \\ *Corresponding author: Trevor Archer, University of \\ Gothenburg, Department of Psychology, Gothenburg, \\ Sweden. Network for Empowerment and Well-being, \\ E-mail: trevor.archer@psy.gu.se \\ www.peertechz.com \\ ISSN: 2455-5460 \\ Keywords: Anorexia nervosa; Positive Affect \\ Negative Affect Schedule; Emotional well-being \\ Negative affect; Sleeping problems; Mood-enhancing \\ drugs; Exercise
}

\section{Research Article \\ Emotional Well-Being in Anorexia Nervosa: Negative Affect, Sleeping Problems, Use of Mood-enhancing Drugs and Exercise Frequency}

\section{Introduction}

Anorexia nervosa (AN) is a severe psychiatric disorder characterized by unrelenting self-starvation and life-threatening weight loss with concomitant brain anomalies as a consequence of malnutrition [1]. This relentless pursuit of starvation is associated with negative physical, emotional, and social consequences [2]. It has been found, that negative affect was increased significantly before loss of control eating, purging, the combination of loss of control eating/and purging, and weighing behavior in a sample of 118 adult females presenting AN. Furthermore, negative affect decreased significantly after the occurrence of these behaviors [3]. Some studies imply that negative affect may offer a critical maintenance mechanism of some AN symptoms. For example, in a study of 116 anorexic women measuring personality, clinical variables, and eating disorder symptoms [4], three subtypes, under-regulated (1), over-regulated (2), and low psychopathology (3), emerged. Underregulated subtypes (1), characterized by high Stimulus-Seeking, Self-Harm, and Oppositional behavior presented higher baseline eating disorder symptoms, lower positive affect, higher negative affect, self-discrepancy, and binge eating. Over-regulated subtypes (2), characterized by high Compulsivity and low Stimulus-Seeking were more likely to have a lifetime obsessive-compulsive disorder diagnosis and showed higher levels of perfectionism, whereas negative affect, positive affect, and self-discrepancy in this group were intermediary between the other subtypes. Low psychopathology subtypes (3), characterized by normative personality, and presented the lowest levels of baseline eating disorder symptoms, co-occurring disorders, and eating disorder behaviors. Nevertheless, in a group of clinically diagnosed Estonian patients presenting eating disorders, compared with healthy volunteers, assessed on Estonian versions of the Eating Disorder Inventory-2, positive affect and negative affect (measured by the Positive Affect and Negative Affect Schedule), and Neuroticism made the largest contribution to Eating Disorder-2 subscales, with Openness to Experience and Conscientiousness (measured by the NEO Personality Inventory), predisposing individuals to eating problems [5]. It has been shown also that patients with AN inhibit expressions of both positive and negative emotions, even after controlling for neuroticism, with higher levels of hostility and neuroticism; they were less aware of their inner thoughts and feelings, "private self-consciousness", and had a heightened awareness of the thoughts and expectations of others, "public selfconsciousness", [6]. Patients presenting eating disorders often report alexithymia, an inability to identify and describe their emotions and affective status $[7,8]$. They show a paucity of words expressing feelings and demonstrate difficulties in identifying and distinguishing feeling of physical sensations. Alexithymia and personal distress seem to predict the vulnerability features of Anorexia Nervosa (AN) with higher levels of personal distress in the latter linked to poor selfregulation and emotional awareness [9].

There is a high comorbidity between AN and anxiety and/or alexithymia disorders [10], moreover, AN patients display certain features similar to those abusing substances/exercise, through a 'narrowed' behavioral repertoire such that weight loss, food intake restriction, and excessive exercise interfere with other activities in a similar fashion to substance/exercise abuse [11]. Further, comorbidity between AN, anxiety, depression and psychoactive substance use has been described [12]. It is likely that the disorder elevates anxiety, depression with stress presenting a predisposing factor to poorer 
health-related quality-of-life and social support [13]. It was observed that patients presenting eating disorders, $\mathrm{AN}$ and bulimia nervosa, showed markedly more alexithymia and a comparable group of female students. Clinical evidence indicates that these patients have major problems with attachment anxiety and negative affect [14]. The core affective processes that evolve into the development and maintenance of AN remain relatively unknown although the contributions of alexithymia suggest an affective basis [15]. It has been observed that AN participants displayed significantly greater implicit positive affect toward pleasant images and significantly greater implicit negative affect toward unpleasant, high-calorie food, and overweight body type images. Recovered participants did not differ significantly from controls on any implicit affect measure [16]. The notion of AN, as a motivated behavioral disorder in which compulsiveness may contribute to the persistence of abnormal eating habits and excessive exercise behaviors, seems warranted in view of evidence implicating anxiety, stress, fear and avoidance learning factors $[17,18]$.

Affect is suggested to be composed of two systems, positive affect and negative affect, which reflect relatively stable cognitive emotional profiles as dispositions or signal sensitivity systems $[19,20]$. Negative affect and emotion among individuals presents a trait associated with both anxiety [21] and depression [22-24]. Conversely, positive affect is negatively associated with depression [24]. Attachment anxiety was associated with eating disorder symptom severity, and this relationship may be mediated by perfectionism and affect regulation strategies [25]. Affective profiles expressing high negative affect have been shown to report a higher degree of stress and a lower degree of coping and control than those with high positive affect [26-28]. Moreover, both adolescents and young and older adults with "Self-destructive" affective profile, i.e. high negative affect and low positive affect, typically report a higher degree of depression than people with any of the other profiles, i.e. "self-fulfilling", "high affective" and "low affective" [19,26]. Negative affect has also been associated with pessimism [29]. Negative affect predicted stress, which in turn predicted general and situational depressiveness [30]. In this context, [24], suggest that positive affect is a dimension that varies from pleasant engagement (e.g., enthusiastic and active) to unpleasant disengagement (e.g., sad and bored), while the negative affect dimension moves from unpleasant engagement (e.g., angry and fearful) to pleasant disengagement (e.g., calm and serene). However, there is evidence that rather than being completely independent, the two affective dimensions might be interrelated in a two-dimensional circumflex model containing not only arousal (vertical axis), but also a valence dimension (horizontal axis). In other words, in order to get a full range of a persons' emotional well-being we need to assess not only high arousal affect, but also to assess the whole range of emotions we humans can experience.

Several implications of decreased emotional well-being are related to mental health concerns such as stress, depression and anxiety which may in turn lead to expressions of physical illhealth. Deterioration in health status, for instance, is associated with reduced levels of emotional and physical well-being [31]. The notion of affectivity presents a confluence of emotions and attributes - psychological, biological and social - that determine individuals' perceptions and experiences of situations [32]. Thus, the purpose of the present study was to examine AN patients' emotional well-being and background health information in comparison to age-matched healthy control subjects.

\section{Materials and Methods}

\section{Participants and procedure}

Ninety female patients (age range: 24 to 42 years) presenting eating disorders at diagnosis but here exclusively AN, with a history of unsuccessful treatment and referred from the Department of General Psychiatry, Sahlgrenska University Hospital (Gothenburg, Sweden) participated in the study. All the patients were ethnic Scandinavians from higher socioeconomic groups and well-educated. They had all undergone further education, following high-school graduation, for at least three years, had affluent-level economic status and uppermiddle social-family backgrounds. They had all been afflicted with the symptoms for over five on arrival at the Anorexia \& Bulimia Clinic for Adults (Sahlgrenska University Hospital), and described themselves as "well-behaved girls". The healthy volunteer participants (i.e., 114 Controls) were selected from a larger sample collected in another study using the same instruments to measure affect [32].

The ethics protocol of the University Hospital Sahlgrenska was applied and maintained. All the patients who were contacted agreed to participate $(\mathrm{N}=90)$. On arrival at the clinic, each patient described her type of eating disorder and completed the Eating Disorder Inventory-2 to measure symptoms of eating disorder and was then given their diagnosis by the presiding staff. Thereafter, were allowed to complete the questionnaire. Specifically, on arrival each patient was met by the respective professional workers, nurse, psychologist, physician, and after a preliminary discussion was asked whether or not she would be willing to complete a questionnaire in order for the health staff to obtain further insights into the AN condition. Following this, each patient received instructions and was allowed 30-45 $\mathrm{min}$ to respond to the instruments.

\section{Instruments}

Background health information. Background variables, such as age, years of education, smoking and drinking habits, pain, use of mood-enhancing drugs and analgesics, and frequency and duration of exercise, etc, were completed according to the description and procedure outlined in [33].

Positive Affect Negative Affect Schedule. This is one of the most commonly used instruments to measure high arousal affect and was developed on the premise that positive affect and negative affect represent two orthogonal independent dimensions: high positive affect versus low positive affect and high negative affect versus low negative affect. The instrument instructs participants to rate to what extent they generally have experienced 20 different feelings or emotions (10 positive affect and 10 negative affect) during the last week, using a 5 -point Likert scale ( $1=$ very slightly, $5=$ extremely). The 10-item positive affect scale includes adjectives such as strong, proud, and interested. The 10-item negative affect scale includes adjectives such as afraid, ashamed, and nervous. In their study [30], reported a Cronbach's alpha of .88 for the positive affect scale and .83 
for the negative affect scale. In the present study the scales had similar internal reliability ( .93 for positive affect and .91 for negative affect).

Emotional Well-Being Scale. This a 16-item questionnaire includes eight items to assess positive feelings and eight items to assess negative feelings. For both the positive and negative items, three of the items are general (e.g., positive, negative) and three per subscale are more specific (e.g., joyful, sad). In contrast to the Positive Affect Negative Affect Schedule, this instrument includes low arousal emotions [34]. In the present study the Cronbach's alpha were .90 for the positive emotion scale and .92 for the negative emotion scale. Two single items form these scales were also used to assess depression and stress.

\section{Results}

AN patients displayed higher levels of negative affect and negative emotions, more stress and depression, greater sleeping problems, pain, and use of analgesics and mood-enhancing drugs, as well as lower levels of positive affect and positive emotions, than the age-matched healthy controls to which they were compared. Nevertheless, the AN patients reported a higher frequency of physical exercise (Table 1).

In order to assess the extent to which the AN condition (as opposed to healthy control condition) may be predicted from participants' emotional well-being (i.e., positive and negative affect, positive and negative emotions, stress, and depression), sleeping problems, pain analgesics, mood-enhancing drugs and exercise frequency, a regression analysis was performed with the former as dependent variable and the latter as independent (predictor) variables. The analysis indicated that the AN condition could be predicted $\left(\mathrm{F}(9,176)=21.33, \mathrm{p}<0.001\right.$, adjusted $\left.\mathrm{R}^{2}=0.512\right)$. The significant predictors were negative affect, sleeping problems, moodenhancing drugs and increased exercise frequency (Table 2). Positive affect, positive and negative emotions, stress and depression, sleeping problems, pain, and analgesics were non-significant.

Table 1: Means and SD ( \pm )Self-reported positive and negative affect, positive and negative emotions, stress and depression levels, sleeping problems, pain, use of analgesics and mood-affecting drugs, and exercise frequency by AN patients (90) and healthy controls $(n=114)$.

\begin{tabular}{|c|c|c|c|}
\hline Factor & $\mathbf{F}$ & AN Patients & $\begin{array}{c}\text { Healthy Age-Matched } \\
\text { Controls }\end{array}$ \\
\hline Positive affect & $111.17^{*}$ & $2.81 \pm 0.82$ & $3.63 \pm 0.71$ \\
\hline Negative affect & $65.04^{*}$ & $3.14 \pm 0.74$ & $2.08 \pm 0.62$ \\
\hline Positive emotions & $27.77^{\star}$ & $2.98 \pm 1.08$ & $3.71 \pm 0.89$ \\
\hline Negative emotions & $36.98^{\star}$ & $3.60 \pm 1.00$ & $2.76 \pm 0.93$ \\
\hline Stress & $84.34^{\star}$ & $4.23 \pm 0.88$ & $2.95 \pm 1.05$ \\
\hline Depression & $40.29^{\star}$ & $3.25 \pm 1.36$ & $2.07 \pm 1.25$ \\
\hline Sleeping problems & $98.87^{\star}$ & $3.84 \pm 1.10$ & $2.39 \pm 0.96$ \\
\hline Pain & $63.69^{*}$ & $3.69 \pm 1.07$ & $2.45 \pm 1.11$ \\
\hline Analgesics & $32.27^{\star}$ & $2.38 \pm 1.22$ & $1.58 \pm 0.78$ \\
\hline Mood-enhancing drugs & $41.81^{*}$ & $2.66 \pm 1.97$ & $1.28 \pm 1.00$ \\
\hline Exercise frequency & $4.60^{\star \star \star}$ & $3.92 \pm 1.18$ & $3.59 \pm 1.07$ \\
\hline
\end{tabular}

${ }^{*} \mathrm{p}<0.001,{ }^{* * *} \mathrm{p}<0.05$. Bonferroni comparisons.
Table 2: Standardized weights from linear regression analysis with AN condition as dependent variable and positive and negative affect, positive and negative emotions, stress, depression, sleeping problems, pain, analgesics, moodenhancing drugs and exercise frequency as independent variables.

\begin{tabular}{|l|c|c|}
\hline Predictor variable & Standardized $\boldsymbol{\beta}$ & Significance \\
\hline Negative affect & 0.289 & $\mathrm{P}<0.01$ \\
\hline Sleeping problems & 0.208 & $\mathrm{P}<0.01$ \\
\hline Exercise frequency & 0.137 & $\mathrm{P}<0.03$ \\
\hline Mood-enhancing drugs & 0.129 & $\mathrm{P}<0.03$ \\
\hline
\end{tabular}

\section{Discussion}

The purpose of the present study was to examine AN patients' emotional well-being and background health information in comparison to age-matched healthy control subjects. The present results were straightforward: AN patients expressed lower levels of positive affect and positive emotions and higher levels of negative affect and negative emotions, together with more stress and depression, greater problems with sleeping and more pain, as well as a higher usage of analgesics and mood-enhancing drug (but not drugs of abuse). The high levels of negative affect and emotions, stress and depression accompanied by low levels of positive affect and emotions are in agreement with previous findings of alexithymia [15]. Nevertheless, the AN patients reported also higher levels of physical exercise frequency, which is contradictory to recent research suggesting that exercise compliance is associated to high levels of positive affect rather than high, or low for that matter, levels of negative affect [35]. Moreover, the regression analysis indicated that the AN condition, i.e. patients having received the diagnosis on the basis of clinic staff interviews and Eating Disorder Inventory-2 responses, was predicted by high levels of negative affect, frequent sleeping problems, higher use of mood-enhancing drugs and high exercise frequency. Taken together, this constellation of harbingers of ill-health implies that, at least in this group of AN patients, the habit of frequently exercising is associated to some degree of comorbidity. In other words, the presence of higher exercise levels is normally linked to health, well-being and positive affect [33,35], however, in the present circumstances it may be likely that elevated exercise contributes an element of 'behavior pathology' to the comorbidity of AN. For instance, the Swedish expression, "well-behaved girl" ("duktig flicka" in Swedish), that the patients used to describe themselves, implies that these individuals probably hid much of their emotional upheaval and problems from their social environment. Nevertheless, these individuals were highly functional despite their condition. These patients tended to handle their emotional upheaval by performing a plethora of 'compensatory' behaviors, such as washing the floor, vacuuming, tidying the apartment, etc.

Although increased levels of physical exercise are associated with better health and well-being [35-38], and better prognosis in psychiatric conditions, the observation of greater exercise frequency in the present study may not necessarily be an entirely positive manifestation. On the one hand, it has been observed that female patients with AN engaged in caloric restriction and excessive exercising prior to clinical treatment had larger hippocampal volumes than healthy comparison women. Following restoration 
of body weight after treatment, AN patients who had engaged in food restriction and excessive exercise prior to treatment had hippocampal volumes similar to that of women with AN who only engaged in caloric restriction. On the other hand, there seems to be a subgroup of high-level AN-exercisers (66\%) with consecutive increased energy requirements [39], contributing to that group of AN 'excessive exercisers'. Groups of AN patients tend to report $57-92 \%$ higher levels of total activity than healthy controls [40], outpatients report more walking and moderate exercise than healthy controls, and inpatients report more walking but less moderate and vigorous activity than all other groups. It has been shown that AN groups had significantly higher drive to exercise and assessed "improving tone" as important whereas health and enjoyment were less important reasons to exercise [40,41]. Furthermore, [42] found that the normalization of body weight in patients with $\mathrm{AN}$ is associated with a preferential distribution of body fat in central regions, which does not, however, seem to influence either eating disorder psychopathology or psychological distress scores. Extent of physical exercise was correlated with trait reward sensitivity, engagement and attentional orienting. However, the finding, in AN patients, of a positive relationship between excessive exercise and obsessive compulsive disorder is unsettling $[43,44]$, have implied that excessive habit formation, the exercise habit, may be construed as a mechanism through which initially rewarding weight loss behavior in AN may develop a compulsiveness over time, with supervised exercise training deemed as 'safe' [45]. AN has been shown to cooccur with obsessive-compulsive disorder and depression [46]. Both Current and lifetime comorbidity of anxiety and depressive disorders with AN has been observed with social phobia, panic disorders, and obsessive-compulsive disorder (OCD) frequently present in AN groups [47].

\section{Limitations of the study}

Whether or not the evidence for a pathological aspect of the excessive exercise shown by AN patients needs to be addressed in further studies that present a specific rationale and assessment. Particular quantitative data is required to demonstrate an eventual pathological aspect to the increased exercise. Similarly, the issue of whether or not AN patients show abnormalities in hedonic and motivational systems requires attention. In the present case, there may be evidence only for simple compulsive behavior. Finally, the present study fails to account for the possibility that increased exercise frequency reflects a compensatory behavior to alleviate anxiety, which is consistent with an anxiolytic action of exercise [48], given the high co-morbidity between AN and anxiety disorders [17].

\section{References}

1. King JA, Geisler D, Ritschel F, Boehm I, Seidel M, et al. (2015) Global cortical thinning in acute anorexia nervosa normalizes following long-term weight restoration. Biol Psychiatry 77: 624-632.

2. Kosmiski L, Schmiege SJ, Mascolo M, Gaudiani J, Mehler PS (2014) Chronic starvation secondary to anorexia nervosa is associated with an adaptive suppression of resting energy expenditure. J Clin Endocrinol Metab 99: 908914.

3. Engel SG, Wonderlich SA, Crosby RD, Mitchell JE, Crow S, et al. (2013) The role of affect in the maintenance of anorexia nervosa: evidence from a naturalistic assessment of momentary behaviors and emotion. J Abnorm Psychol 122: 709-719.

4. Lavender JM, Wonderlich SA, Crosby RD, Engel SG, Mitchell JE, et al. (2013) Personality-based subtypes of anorexia nervosa: examining validity and utility using baseline clinical variables and ecological momentary assessment. Behav Res Ther 51: 512-517.

5. Podar I, Hannus A, Allik J (1999) Personality and affectivity characteristics associated with eating disorders: a comparison of eating disordered, weightpreoccupied, and normal samples. J Pers Assess 73: 133-147.

6. Forbush K, Watson D (2006) Emotional inhibition and personality traits: a comparison of women with anorexia, bulimia, and normal controls. Ann Clin Psychiatry 18: 115-121.

7. Courty A, Godart N, Lalanne C, Berthoz S (2015) Alexithymia, a compounding factor for eating and social avoidance symptoms in anorexia nervosa. Compr Psychiatry 56: 217-228.

8. Guillen V, Santos B, Munoz P, Fernandez de Corres B, Fernandez E, et al. (2014) Toronto alexithymia scale for patients with eating disorder: [corrected] performance using the non-parametric item response theory. Compr Psychiatry 55: 1285-1291.

9. Beadle JN, Paradiso S, Salerno A, McCormick LM (2013) Alexithymia emotional empathy, and self-regulation in anorexia nervosa. Ann Clin Psychiatry 25: 107-120.

10. Brand-Gothelf A, Leor S, Apter A, Fennig S (2014) The impact of comorbid depressive and anxiety disorders on severity of anorexia nervosa in adolescent girls. J Nerv Ment Dis 202: 759-762.

11. Barbarich-Marsteller NC, Foltin RW, Walsh BT (2011) Does anorexia nervosa resemble an addiction? Curr Drug Abuse Rev 4: 197-200.

12. Jordan J, Joyce PR, Carter FA, Horn J, McIntosh VV, et al. (2003) Anxiety and psychoactive substance use disorder comorbidity in anorexia nervosa or depression. Int J Eat Disord 34: 211-219.

13. Nekouei ZK, Doost HT, Yousefy A, Manshaee G, Sadeghei M (2014) The relationship of alexithymia with anxiety-depression-stress, quality of life, and social support in coronary heart disease (A Psychological Model). J Educ Health Promot 23: 68.

14. Harney MB, Fitzsimmons-Kraft EE, Maldonado CR, Bardone-Cone AM (2014) Negative affective experiences in relation to stages of eating disorder recovery Eat Behav 15: 24-30.

15. Lundblad S, Hansson B, Archer T (2014) Affect-group intervention for alexithymia in eating disorders. Int J Emerg Ment Heal Hum Resourc 17: 219223.

16. Spring VL, Bulik CM (2014) Implicit and explicit affect toward food and weight stimuli in anorexia nervosa. Eat Behav 15: 91-94.

17. Guarda AS, Schreyer CC, Boersma GJ, Tamashiro KL, Moran TH (2015) Anorexia nervosa as a motivated behavior: Relevance of anxiety, stress, fear and learning. Physiol Behav. pii: S0031-9384(15)00202-4.

18. Wable GS, Min JY, Chen YW, Aoki C (2015) Anxiety is correlated with running in adolescent female mice undergoing activity-based anorexia. Behav Neurosci 129: 170-182.

19. Archer T, Kostrzewa RM, Beninger RJ, Palomo T (2008) Cognitive symptoms facilitatory for diagnoses in neuropsychiatry. Neurotox Res 14: 205-225.

20. Garcia D (2012a) The affective temperaments: differences between adolescents on the big five and Cloninger's psychobiological model of personality. J Happiness Stud 13: 999-1017.

21. Brytek-Matera A, Schiltz L (2009) A cross-cultural examination of the relationship between negative mood states, body image and eating disorders. Bull Soc Sci Med Grand Duche Luxemb 2009:11-25.

22. Ebesutani C, Okamura K, Higa-McMillan C, Chorpita BF (2011). A psychometric analysis of the positive and negative affect schedule for 
children-parent version in a school sample. Psychologi- cal Assessment 23: 406-416

23. Luten AG, Ralph JA, Mineka S (1997) Pessimistic attribu- tional style: is it specific to depression versus anxiety versus negative affect? Behavioral Research and Therapy 35: 703-719.

24. Watson D, Clark LA, Carey G (1988) Positive and negative affectivity and their relation to anxiety and depressive disorders. J Abnorm Psychol 97: 346353.

25. Tasca GA, Balfour L (2014) Attachment and eating disorders: a review of current research. Int J Eat Disord 47: 710-717.

26. Archer T, Adrianson L, Plancak A, Karlsson E (2007) Influ- ence of affective personality on cognition-mediated emotion proc- essing: Need for empowerment. European Journal of Psychiatry 21: 248-262.

27. George JM, Brief AP (1992) Feeling good-doing good: A conceptual analysis of the mood at work-organizational spontaneity relationship. Psychological Bulletin 112: 310-329.

28. Spector PE, O'Connell BJ (1994) The contribution of personal- ity traits negative affectivity, locus of control and Type A to the subsequent reports of job stressors and job strains. Journal of Occu- pational and Organizational Psychology 67: 1-12.

29. Marshall GN, Wortman C, Kusulas JW, Hervig LK, Vickers Jr, et al. (1992) Distinguishing optimism from pessimism: Relations to fundamental dimensions of mood and personality. Journal of Per- sonality and Social Psychology 62: 1067-1074.

30. Lindahl M, Archer T (2013) Depressive expression and anti-depressive protection in adolescence: Stress, positive affect, motivation and self-efficacy. Psychology 4: 495-505

31. Doll HA, Petersen SEK, Stewart-Brown SL (2012) Obesity and Physical and Emotional Well-Being: Associations between Body Mass Index, Chronic Illness, and the Physical and Mental Components of the SF-36 Questionnaire. Obes Res 8: 160-170.

32. Sailer U, Rosenberg P, Nima AA, Gamble A, Gärling T, Archer T, (2014) A Happier and Less Sinister Past, a More Hedonistic and Less Fatalistic Present and a more Structured Future: Time Perspective and Well-Being. PeerJ 2: e303.

33. Palomo T, Beninger RJ, Kostrzewa RM, Archer T (2008) Affective status in relation to impulsive, motor and motivational symptoms: personality, development and physical exercise. Neurotox Res 14: 151-168.
34. Garcia D (2012b) Intensity vs. Frequency: Personality and the Affective Component of Subjective Well-Being. 120th Annual Convention of the American Psychological Association. Orlando, Florida, USA.

35. Garcia D, Archer T (2014) Positive affect and age as predictors of exercise compliance. PeerJ 2e: 694

36. Archer T (2014) Health benefits of physical exercise for children and adolescents. J Novel Physiother 4: 2.

37. Archer T, Garcia D (2014) Physical exercise influences academic performance and well-being in children and adolescents. Int J School Cogn Psychol.

38. Archer T, Garcia D (2015) Exercise and dietary restriction for promotion of neurohealth benefits. 7

39. Zipfel S, Mack I, Baur LA, Hebebrand J, Touyz S, et al. (2013) Impact of exercise on energy metabolism in anorexia nervosa. J Eat Disord 1: 37.

40. Keyes A, Woerwag-Mehta S, Bartholdy S, Koskina A, Middleton B, et al. (2015) Physical activity and the drive to exercise in anorexia nervosa. Int J Eat Disord 48: 46-54

41. Sternheim L, Danner U, Adan R, van Elburg A (2015) Drive for activity in patients with anorexia nervosa. Int J Eat Disord 48: 42-45

42. El Ghoch M, Milanese C, Calugi S, Pellegrini M, Battistini NC, et al. (2014) Body composition, eating disorder psychopathology, and psychological distress in anorexia nervosa: a longitudinal study. Am J Clin Nutr 99: 771-778.

43. Davis C, Kaptein S (2006) Anorexia nervosa with excessive exercise: a phenotype with close links to obsessive-compulsive disorder. Psychiatry Res 142: 209-217.

44. Godier LR, Park RJ (2014) Compulsivity in anorexia nervosa: a transdiagnostic concept. Front Psychol. 5: 778

45. Ng LW, Ng DP, Wong WP (2013) Is supervised exercise training safe in patients with anorexia nervosa? A meta-analysis. Physiotherapy 99: 1-11.

46. Simpson HB, Wetterneck CT, Cahill SP, Steinglass JE, Franklin ME, et al. (2013) Treatment of obsessive-compulsive disorder complicated by comorbid eating disorders. Cogn Behav Ther 42: 64-76.

47. Godart N, Berthoz S, Rein Z, Perdereau F, Lang F, et al. (2006) Does the frequency of anxiety and depressive disorders differ between diagnostic subtypes ofanorexia nervosa and bulimia? Int J Eat Disord 39: 772-778

48. LeBouthillier DM, Asmundson GJ (2015) A Single Bout of Aerobic Exercise Reduces Anxiety Sensitivity But Not Intolerance of Uncertainty or Distress Tolerance: A Randomized Controlled Trial. Cogn Behav Ther 15: 1-12.

Copyright: (c) 2015 Lundblad S, et al. This is an open-access article distributed under the terms of the Creative Commons Attribution License, which permits unrestricted use, distribution, and reproduction in any medium, provided the original author and source are credited. 